\title{
Numerical Simulation of Aqueous Flow in Laser Iridotomy
}

\author{
Bin Chen, Yibo Zhao, Dong Li \\ State Key Laboratory of Multiphase Flow in Power Engineering, Xi'an Jiaotong University \\ Xi'an, Shanxi, China \\ chenbin@mail.xjtu.edu.cn
}

\begin{abstract}
Primary angle-closure glaucoma (PACG) is a major cause of blindness worldwide, with a particularly high prevalence in Asian populations. Laser iridotomy (LI) has been the standard therapeutic modality for the treatment of PACG to avoid blindness. However, physiological mechanism of LI has not been fully understood, due to the complex structure of the eyeball, the fluidity of aqueous, and the limitation of detecting equipment. It will increase the difficulty of surgery and the probability of complications. Based on the above reason, numerical analysis was conducted to investigate the aqueous humor flow under different physiological structures before and after laser surgery. It will be helpful to explore the pathological cause of primary angle-closure glaucoma, prevent the complications of laser iridotomy, and provide new ideas for clinical guidance. In this paper, the main flow domain of aqueous humor was modelled by three kinds of structure of eyeball according to the physiological theory of the treatment process. They were normal eyeball, eyeball with the physiological structure of shallow anterior chamber depth and narrow angle and eyeball after laser iridotomy treatment. The finite volume method was used to discretize the computational model area. The flow of aqueous humor is simulated. The results showed that, 1) the geometric model of physiological structure of shallow anterior chamber depth was very important for theoretical study of glaucoma. When the depth of anterior chamber decreased from $2.8 \mathrm{~mm}$ to $2.0 \mathrm{~mm}$, the maximum velocity of natural convection doubled, and the pressure difference of shallow anterior chamber increased by 20\%. 2)Pupil block increases intraocular pressure sharply, which is equivalent to thousands of times the normal pressure, causing damage to intraocular tissues. 3)Laser iridectomy can effectively reduce the intraocular pressure caused by pupil block, but the velocity of aqueous humor after operation is 40 times of normal speed, and the increase of corneal shear stress leads to corneal damage.
\end{abstract}

Keywords: Primary angle-closure glaucoma, Laser iridotomy, Aqueous humor flow, Numerical analysis.

\section{Introduction}

Primary angle-closure glaucoma (PACG) is a major cause of blindness worldwide, with a particularly high prevalence in Asian populations. It is estimated that the number of people with PACG worldwide will increase to 23.4 million in 2020 , $76.7 \%$ of whom will be Asian ${ }^{[1]}$. It refers to the peripheral iris blocking the trabecular meshwork or permanent adhesion with it, then the angle of the anterior chamber is closed, and the aqueous outflow obstructed, causing intraocular pressure increased beyond the degree of tolerance of the eyeball. It will lead to optic nerve atrophy, visual field shrinkage, visual impairment and even blindness.

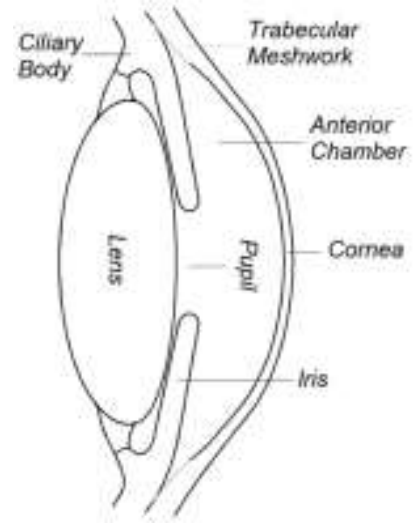

Fig. 1: Cyclicprocess of aqueous humor ${ }^{[2]}$. 
The pathogenesis of glaucoma is closely related to the abnormal flow of aqueous humor. The aqueous humor (AH) inflates the globe of the eye with maintaining the intraocular pressure. In addition, it provides the required nutrition for the avascular ocular tissues, posterior cornea, trabecular meshwork, lens, and anterior vitreous. The anterior chamberis a space inside the eye between the iris and the innermost surface of cornea that is filled with aqueous humor (AH). In Fig. 1, the ciliary body secretes the AH into the posterior chamber at a rate of approximately $1.5-3.0 \mu \mathrm{L} / \mathrm{min}$, and then this fluid flows through the narrow cleft between the lens and the iris and escapes through the pupil into the anterior chamber. Then, the AH finally drains out of the eye via the trabecular meshwork, which results in a residence time of approximately 100 minutes $^{[2]}$.

Only when the anterior chamber is in a certain depth, can the aqueous humor be discharged smoothly, and the intraocular pressure can be maintained within the normal range. However, the anterior chamber of angle-closure glaucoma is usually shallow, which is not conducive to the discharge of aqueous humor, and easily causes increased intraocular pressure. In addition, pupil block is the direct cause of elevated intraocular pressure in angle-closure glaucoma. Pupil block refers to the close contact between the pupil and lens, which blocks aqueous humor flowing from posterior chamber into anterior chamber through the pupil. So aqueous humor can not flow out and accumulates in the posterior chamber, leading to elevated intraocular pressure.

Laser iridectomy(LI) is the most common surgical method for primary angle-closure glaucoma. The iris is perforated by laser photothermal effect. So the aqueous humor can enter the anterior chamber by the perforated position. By eliminating the pupil blocking effect, the adhesion of anterior chamber angle is reduced, and the aqueous humor can be expelled smoothly at the trabecular meshwork to alleviate the increase of intraocular pressure ${ }^{[3]}$.

Although laser iridotomy is non-invasive and has no risk of intraocular hemorrhage or wound leakage, there are still a number of complications after surgery, including iris atrophy, corneal thermal injury and mechanical injury, pupillary ectopic deformation, retinal thermal injury, lens opacity, etc; In addition, the pathogenesis of glaucoma remains unclear. Intraocular pressure is the only known risk factor for the development of glaucomatous optic neuropathy. Studying the intraocular aqueous flow plays an important role in controlling the pathogenesis of high intraocular pressure glaucoma; But in clinical medicine, the physiological mechanism and pathological changes of the eye can not be explored by anatomical analysis of the animal's eyeballs or the intraocular tissues removed from the lesions. The mechanical properties of the wall around the flow field, the velocity, pressure and temperature of the flow field can not be measured directly.

Based on the above reasons, it is necessary to simulate the flow characteristics of aqueous humor in the eyeball by numerical modeling. The characteristics of aqueous humor field, the micro-flow state of intraocular fluid, thermodynamic characteristics, and the regulation mechanism of aqueous humor produced by ciliary trabecular meshwork and other organs can be dynamically studied and observed.

For a long time, the numerical study on the factors affecting the flow of aqueous humor has focused on three aspects:

(1) Analysis of the flow mechanism of aqueous humor: The aqueous humor (AH) flow in the anterior chamber (AC) due to saccadic movements is investigated by Abouali, et $\mathrm{a}^{[4]}$. Ooi ${ }^{[5]}$ proposed various hypotheses to describe the cause of the aqueous humor flow. These hypotheses include the thermally induced flow, the production and drainage of aqueous humor, the mixing and stirring during rapid eye movement (REM) sleep and phakodenesis. Yamamoto and Yasuaki ${ }^{[6]}$ calculated the shear stress exerted on the corneal endothelial cellsin anterior chambers of different depths $(2.8$, $1.8,1.5$, and $1.0 \mathrm{~mm}$ ) by a computational fluid dynamics program. (2) Study on the resistance of aqueous humor outflow: Avtar and Srivastava ${ }^{[7]}$ developed a mathematical model which treated the Schlemm's canal as a porous compliant channel that is held open by the trabecular meshwork and studied the aqueous fluid pressure and flow profiles in the proposed model. Crowder and Ervin ${ }^{[8]}$ presented pressure computations for different flow rates of the aqueous humor, viscosity of the aqueous humor, and permeability of the trabecular meshwork. (3) Fluid-solid coupling system calculation of iris and aqueous humor: Huang ${ }^{[9]}$ investigated the response of iris contour and curvature to mechanical interaction between iris and intraocular structure due to accommodative microfluctuations. Wang, Wenjia ${ }^{[10]}$ studied the effect of IOP, localization, and temperature on the flow of the aqueous humor and the deformation of iris interacted by aqueous humor fluid flow.

For a long time, many scholars have devoted themselves to the study of influencing factors of aqueous humor flow.However, few studies have been done on the pathogenesis of high intraocular pressure glaucoma and the treatment process of laser iridectomy. The changes of parameters and characteristics of aqueous humor field in the whole process 
of angle-closure glaucoma and laser iridectomy are not discussed. The pertinent study of changes in aqueous humor field throughout the onset and treatment process will play a crucial role in the study of physiopathological process of high intraocular pressure glaucoma and the optimization of surgical protocols.

The purpose is to explore the pathogenesis of high intraocular pressure glaucoma and optimize the operation plan of laser iridectomy. The innovation of this paper is to study the aqueous humor flow from the onset of glaucoma to the end of laser treatment, and to deeply explore the changes of parameters and the effects on various tissues in the eyeball. As far as we know, no previous research has been conducted in such studies.

In order to better study the whole process from onset to end of treatment, we have made the following improvements on the mathematical model: 1) The anterior chamber of the patients with angle-closure glaucoma is usually shallow, which is not conducive to the discharge of aqueous humor. However, the geometric models established in the literature on the flow characteristics of aqueous humor are normal eyeballs (anterior chamber depth is $2.8 \mathrm{~mm} 3.0 \mathrm{~mm}$ ), which did not study the physiological structure of shallow anterior chamber of glaucoma $(\sim 2.0 \mathrm{~mm})$. 2) The existing laser iridotomy models believe that the natural convection driven flow field intensity in the anterior chamber is two orders of magnitude larger than the aqueous flow velocity through the pupil, so neither consider the aqueous humor flow in the posterior chamber nor the process of aqueous humor circulation by inflow and outflow pathways. But in the process of pupil blockade, the gap between lens and iris gradually narrows, which will cause the pupil flow speed to accelerate sharply. Therefore, the influence of the velocity drive of aqueous inlet on the flow field should be considered.

In this paper, the main flow domain of aqueous humor (anterior and posterior chambers of eyeball) was modelled by three kinds of structure of eyeball according to the physiological theory of the treatment process. They were normal eyeball, eyeball with the physiological structure of shallow anterior chamber depth and narrow angle and eyeball after laser iridotomy treatment. The finite volume method was used to discretize the computational model area. The flow of aqueous humor under primary angle-closure glaucoma conditions is simulated. It will be helpful to explore the pathological cause of primary angleclosure glaucoma, prevent the complications of laser iridotomy, and provide new ideas for clinical guidance.

\section{Analysis and modelling}

\subsection{Simplified eyeball model}

Since we use the main flow area of aqueous humor as the research model, we mainly consider the anterior part of the eyeball, that is, the area from the intraocular lens to the cornea. The boundaries of the model are the cornea, lens, vitreous, iris, ciliary body and trabecular meshwork.

According to the description of glaucoma incidence and laser surgery process, four types of eyeball structure models were established. Fig. 2 shows the eyeball structure models under four different physiological states. Fig. 2 (a) is the eyeball with normal physiological function, Fig. 2 (b) is the eyeball with shallow chamber angle and narrow chamber angle (the structural premise of glaucoma), Fig. 2 (c) is the eyeball model after laser iridectomy, aqueous humor flows into the anterior chamber from the laser hole, and pupil block is relieved.
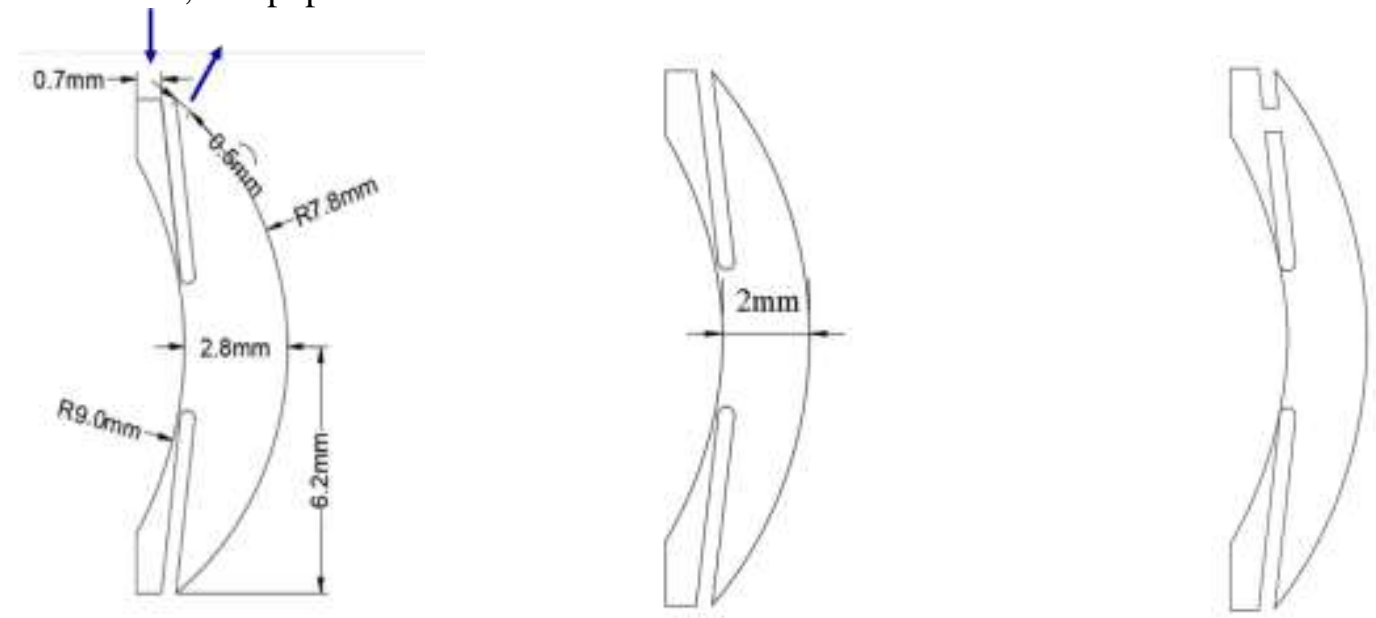

(a) normal eyeball (b) eyeball of shallow anterior chamber (c) eyeball after laser iridotomy treatment

Fig. 2: Cyclicprocess of aqueous humor. 
In this model, natural convection is considered. The direction of gravity is the negative direction along the $\mathrm{Y}$ axis, and $g=9.81 \mathrm{~m} / \mathrm{s}^{2}$. The iris is a flat disc structure. The posterior surface of cornea and the anterior capsule of lens are defined as a dome structure. In this paper, the curvature radius of cornea is $7.8 \mathrm{~mm}$, the curvature radius of lens is $9 \mathrm{~mm}$, the height of the whole model is $12.4 \mathrm{~mm}$, the width of ciliary body entrance is $0.7 \mathrm{~mm}$, and the width of trabecular meshwork outlet is $0.5 \mathrm{~mm}$. The distance between lens center and cornea center (anterior depth) in normal eyeball (a) is $2.8 \mathrm{~mm}$, which can represent the size of normal eyeball. In order to better explore the pathogenesis of angle-closure glaucoma and the principle of surgical treatment,(b), (c) take the physiological structure of shallow anterior chamber (depth $=2.0 \mathrm{~mm})$ as the research object. In the geometric model, aqueous humor secreted by the ciliary body enters the posterior chamber at a constant rate of 1.5-3.0 $\mu \mathrm{L} / \mathrm{min}$, passes through the pupil between the lens and the iris, enters the anterior chamber, and finally leaves the anterior chamber through the trabecular meshwork. In 2 (c), there is a cylindrical hole in the iris, which are used to simulate the laser hole on the iris after laser surgery. The diameter of the laser holes is $0.56 \mathrm{~mm}$, and the position of the holes is 12 points and $5 \mathrm{~mm}$ away from the pupil axis ${ }^{[11]}$.

\subsection{Control equation}

Aqueous humor is regarded as incompressible Newtonian viscous fluid, and the continuity equation is

$$
\frac{\partial \rho}{\partial t}+\nabla \cdot(\rho \vec{v})=0
$$

The Navier-Stokes equation is

$$
\frac{\partial \vec{v}}{\partial t}+(\vec{v} \cdot \nabla) \vec{v}+\frac{1}{\rho} \nabla p-g-\mu \nabla^{2} \vec{v}=0
$$

where $\rho$ is fluid density, $t$ represents time, $\vec{v}$ is velocity vector, $p$ is static pressure, $\mathrm{g}=9.8 \mathrm{~m} / \mathrm{s}^{2}$ is gravitational acceleration, and $\mu$ is fluid kinematic viscosity.

Because of the thermal convection between the cornea and the external environment, there is a temperature difference between the cornea and the human nucleus temperature $(37 \mathrm{C})$, so there is a buoyancy-driven natural convection in the anterior chamber. The above equation is coupled to the energy equation.

$$
\frac{\partial T}{\partial t}+(\vec{v} \cdot \nabla) T-\frac{k}{\rho c_{\mathrm{p}}} \nabla^{2} T-S_{T}=0
$$

Where $T$ is the temperature, $K$ is the thermal conductivity of the fluid, $c_{\mathrm{p}}$ is the specific heat capacity of the fluid, $S_{T}$ is the viscous dissipation term. In this paper, we neglect the heat generation caused by mechanical energy dissipation.

Because the main driving force in anterior chamber is natural convection caused by temperature difference, Boussinesq approximation is often used to deal with buoyancy term caused by temperature difference in numerical calculation of natural convection heat transfer in enclosed space. The Boussinesq approximation is very accurate for liquids experiencing very small temperature variations.

It consists of three parts: 1. Viscous dissipation in fluid is neglected; 2. Physical properties are constant except density; 3. For density, only the terms related to mass force in momentum equation are considered, and the densities in other items are also constant.This paper assumes that the density of aqueous humor has a small linear dependence on temperature and is independent of pressure

$$
\rho=\rho_{\mathrm{C}}\left[1-\alpha\left(T-T_{\mathrm{C}}\right)\right]
$$

$\alpha$ is the coefficient of volume expansion.

With the Boussinesq approximation, the governing equation can be simplified to: 


$$
\begin{gathered}
\nabla \cdot \vec{v}=0 \\
\rho \vec{v} \cdot \nabla \vec{v}+\nabla p-\rho g \alpha\left(T-T_{\mathrm{C}}\right)-\rho \mu \nabla^{2} \vec{v}=0 \\
\rho c_{\mathrm{p}} \vec{v} \cdot \nabla T-k \nabla^{2} T=0
\end{gathered}
$$

The physically realistic and mathematically consistent boundary conditions for the steady-state problem are prescribed as follows: The outer surface of the cornea has thermal convection with the external environment

$$
-k \frac{\partial T}{\partial n}=h_{0}\left(T-T_{0}\right)
$$

where $h_{0}$ is the thermal convection coefficient of the cornea and the surrounding environment, $T_{0}$ is the ambient temperature.

It is assumed that the eyeball is embedded in a network filled with blood vessels, and its temperature is the same as the core temperature (blood temperature) of the human body due to the circulation of blood inside the capillaries. Therefore, it is assumed that the lens and the iris adopt the first type of boundary condition, the temperature is $37^{\circ} \mathrm{C}$.

Both the aqueous humor inlet and the exit trabecular mesh are considered rigidly permeable boundaries, and the tangential velocity of $\mathrm{AH}$ at these boundaries is set to zero (no slip). The rate of aqueous humor is not affected by intraocular pressure, thus ensuring the supply of aqueous humor inlet flow. In the eyeball model of normal aqueous circulation, the aqueous flow rate was $2.4 \mu \mathrm{l} / \mathrm{min}$. In order to ensure that the flow rate is constant, the normal eyeball (a) and the eyeball with the shallow depth anterior chamber (b) have adopted the velocity boundary conditions. The velocities are calculated according to the import and export area. The inlet velocity of the ciliary body in (a) and (b) was $v=1.990 \mu \mathrm{m} / \mathrm{s}$, and the outlet velocity of the trabecular meshwork was $v=2.910 \mu \mathrm{m} / \mathrm{s}$.

Yamamoto $^{[11]}$ obtained the $v_{\max }$ of the forward aqueous streaming through the LI window in eyes with the diameter of the LI window at $0.56 \mathrm{~mm}$ was $9.39 \mathrm{~mm} / \mathrm{s}$, determined by the particletracking velocimetry technique. So the entrance condition of physiological model (c) is $v=9.39 \mathrm{~mm} / \mathrm{s}$ along the normal direction. Outflow condition is adopted for flow export.

Table 1 summarizes the values for the physical parameters used in the model.

Table 1: physical parameters ${ }^{[5,6,8,9]}$.

\begin{tabular}{cc}
\hline Physical parameter & Parameter value \\
\hline Specific heat capacity $C / \mathrm{J}(\mathrm{kg} \cdot \mathrm{K})^{-1}$ & 4178 \\
Density $\rho / \mathrm{kg} \cdot \mathrm{m}^{-3}$ & 1000 \\
Thermal conductivity $\mathrm{k} / \mathrm{W} \cdot(\mathrm{m} \cdot \mathrm{K})^{-1}$ & 0.58 \\
Dynamic viscosity $\mu / \mathrm{Pa} \cdot \mathrm{s}$ & $7.5 \times 10^{-4}$ \\
Volume expansion coefficient $\alpha / \mathrm{K}^{-1}$ & $3.1 \times 10^{-4}$ \\
Aqueous humor flow $Q / \mu 1 \cdot \mathrm{min}^{-1}$ & 2.4 \\
Corneal convection coefficient $h_{0} / \mathrm{W} \cdot\left(\mathrm{m}^{2} \cdot \mathrm{K}\right)^{-1}$ & 10 \\
\hline
\end{tabular}

The number of grids used in the simulation is 20,000 to 30,000 . In order to meet the requirements of calculation accuracy, The minimum gap $0.5 \mu \mathrm{m}$ used in the simulation is also divided into 5 layers of grid to ensure the correctness of the calculation results. The flow region is solved by Fluent, the finite volume method is used to discretize the calculation model area. Since the Rayleigh number $(R a)$ is $<10^{6}$, the flow of aqueous humor is approximated as laminar flow. Because the model is relatively complex, the momentum and energy equations are discrete using the second-order upwind style, and the pressure and velocity are based on the SIMPLE algorithm. For all simulations, careful grid independence testing has been performed and the results are authentic 


\section{Results and discussion}

In this part, the numerical model was firstly verified, and then the glaucoma pathogenesis and the changes of the flow field parameters before and after the laser iridotomy.

\subsection{Model correctness verification}

In this section, the validation of the numerical model is presented. No experimental data is available for the flow in the AC due to eye movements. Furthermore, the exact solution of the governing equations cannot be found because of the complexity of the boundary. Therefore, the numerical model is validated in comparison with the theoretical results of Heys ${ }^{[12]}$.

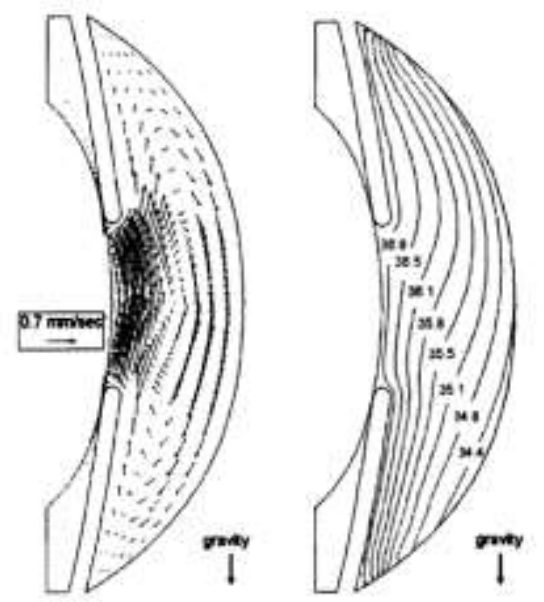

Fig. 3: Flow field characteristics of Heys.
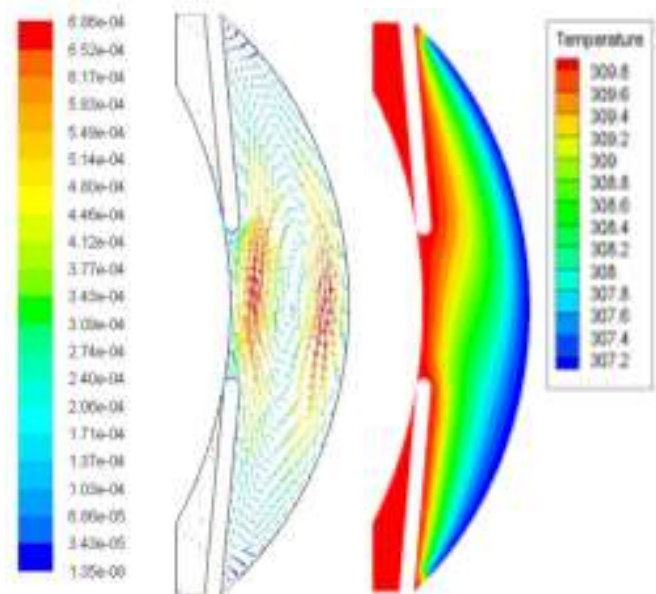

Fig. 4: Flow field characteristics of this paper.

In this section, the pressure, velocity and temperature field of the normal eye modelare calculated. The corneal temperature was $34^{\circ} \mathrm{C}$, the inlet $\mathrm{v}=1.990 \mu \mathrm{m} / \mathrm{s}$, the outlet $\mathrm{v}=2.910 \mu \mathrm{m} / \mathrm{s}$, the gap between lens and iris is $5 \mu \mathrm{m}$. In the Heys study, the flow field and temperature field are shown in Fig. 3. The flow parameters of the model are shown in Fig. 4. The entire velocity vector is consistent with that of Heys in the literature. The peak velocity in the velocity field is 0.684 $\mathrm{mm} / \mathrm{s}$, which is basically the same as the peak temperature of Heys of $0.7 \mathrm{~mm} / \mathrm{s}$. The temperature field is also asymmetric under the action of gravity, which is consistent with the situation of this study, which proves the correctness of this study.

\subsection{Analysis of flow mechanism}

There are two driving forces for aqueous humor flow, one is forced convection caused by inlet velocity, and the other is natural convection caused by temperature difference between cornea and internal tissue of the eyeball. Usually, the aqueous flow field is the result of two kinds of interactions. In this section, the two effects are studied separately to explore the effects of different effects on the flow characteristics of aqueous humor, and to understand the flow mechanism of aqueous humor.

Figure 5 shows the flow of aqueous humor driven by temperature difference, $\Delta T=3{ }^{\circ} \mathrm{C}$, inlet $v=0 \mu \mathrm{m} / \mathrm{s}$, outlet outflow conditions; Figure 6 is the flow velocity of the inlet only, $\Delta T=0^{\circ} \mathrm{C}$ (corneal temperature $37^{\circ} \mathrm{C}$ ), inlet $v=1.990 \mu \mathrm{m} / \mathrm{s}$, outlet $v=2.910 \mu \mathrm{m} / \mathrm{s}$; Figure 7 is the flow characteristics of aqueous humor under the combined effect of temperature difference and inlet velocity.

In Fig. 5, the aqueous humor has a relatively low density inside the eyeball where the temperature is high, and the density is relatively high where close to the cornea, and the difference in density produces a vertical buoyancy. In this way, under the driving force of buoyancy, natural convection is formed, which is a clockwise flow in the two-dimensional section. The pressure distribution only slightly decreases in the direction of gravity, and the entire pressure distribution does not change substantially. In Fig. 6, there is only forced convection driven by the inlet velocity. The sudden contraction of the crosssectional area causes a sharp increase in the flow velocity at the gap between the iris and the lens, and the maximum velocity is $0.3 \mathrm{~mm} / \mathrm{s}$. In the pressure distribution, only the pressure difference between anterior and posterior chambers are significantly different $(\sim 4 \mathrm{~Pa})$. 
Figure 7 shows the aqueous flow under the combined action of forced convection and natural convection. In the velocity vector diagram, the velocity of the peak area in the anterior chamber is $0.686 \mathrm{~mm} / \mathrm{s}$, which bigger than the peak velocity generated by the temperature drive $(0.61 \mathrm{~mm} / \mathrm{s})$. The increase of the velocity is due to the coupling of forced convection on the basis of natural convection. The pressure distribution is the same as that when only the inlet speed is driven, indicating that the dynamic pressure change caused by the velocity does not affect the pressure distribution. In Fig. 7, the temperature field is slightly tilted compared with Fig. 5, and it is known that the larger the flow velocity in the anterior chamber, the greater the inclination of the temperature field.

It can be seen from the analysis that the pressure difference is determined by the inlet velocity in the aqueous flow; the flow field in the anterior chamber is determined by the interaction of natural convection and forced convection, but the influence of natural convection is relatively large.
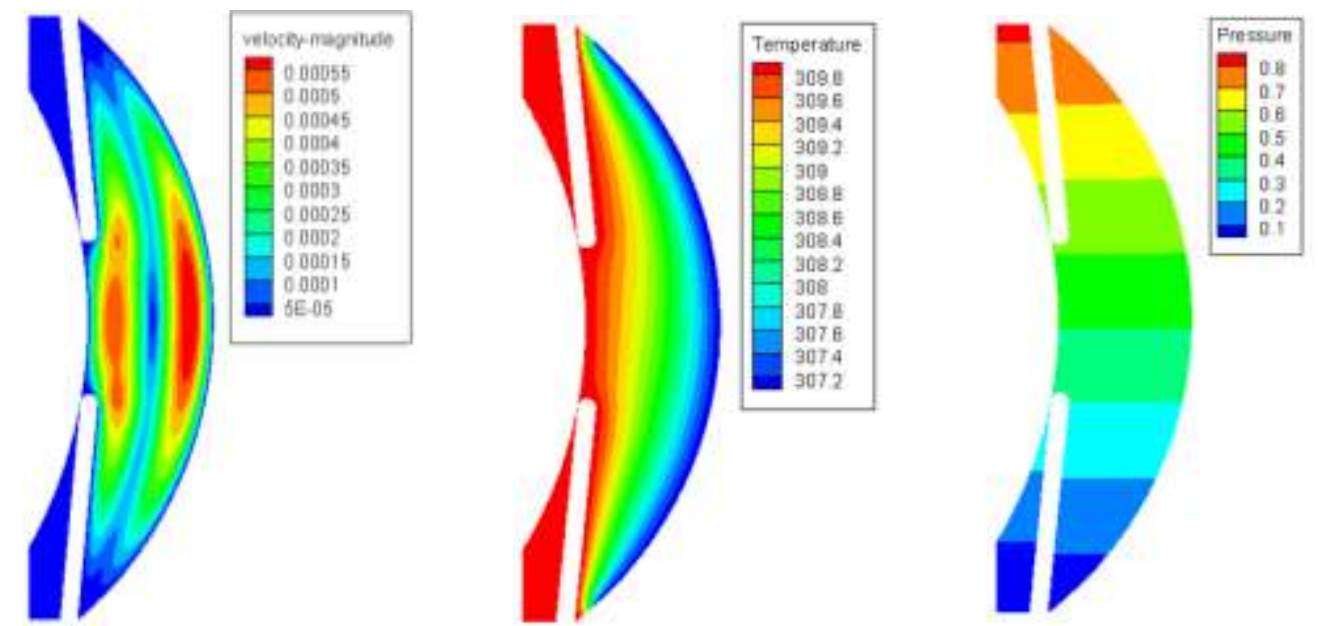

Fig. 5: the flow of aqueous humor driven by temperature difference.
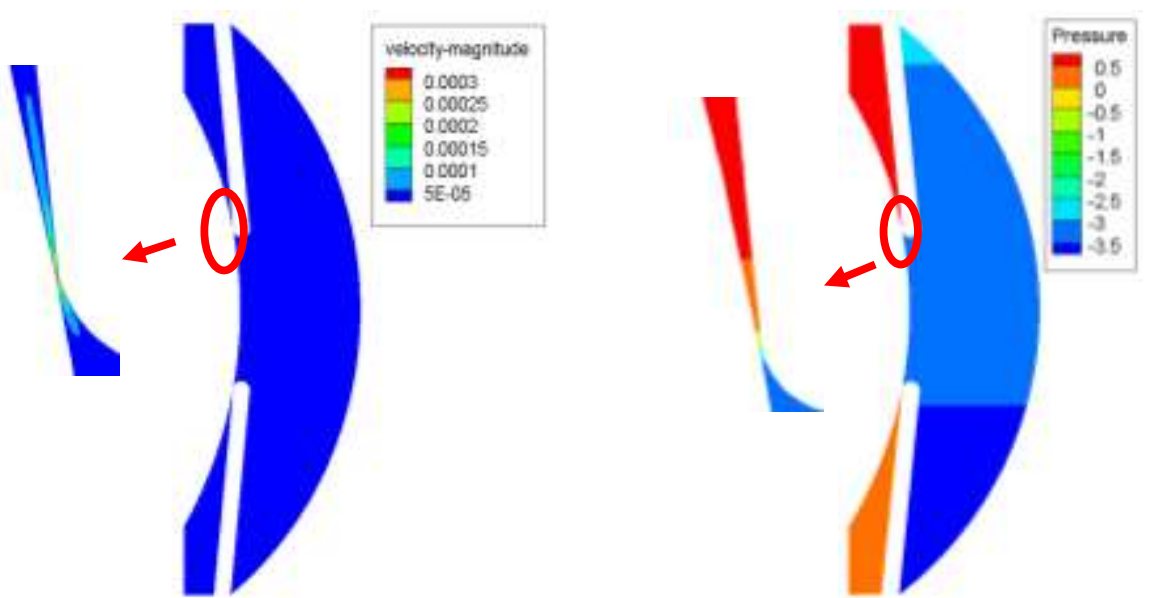

Fig. 6: the flow of aqueous humor driven by inlet velocity. 

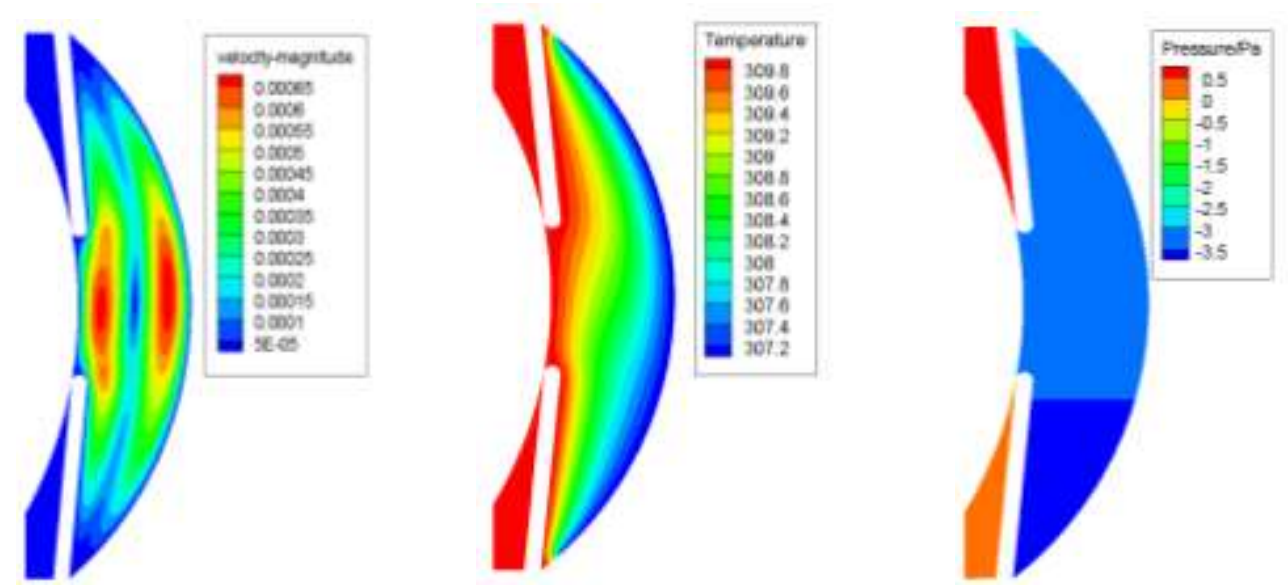

Fig. 7: the flow of aqueous humor driven by inlet velocity and temperature difference(normal eye).

\subsection{Analysis of flow mechanism}

Previous studies on aqueous humor flow theory have focused on normal eyeballs. The structural premise of glaucoma is the existence of anterior chamber with shallow depth. Therefore, the flow field in normal eyeball (anterior chamber depth $2.8 \mathrm{~mm}$ ) and non-onset glaucoma (anterior chamber depth $2.0 \mathrm{~mm}$ ) with shallow depth chamber physiological structure was compared in order to explore the influence of the accuracy of physiological structure model on numerical simulation.

In this section, the corneal temperature is $34^{\circ} \mathrm{C}$, the entrance $v=1.990 \mu \mathrm{m} / \mathrm{s}$, the exit $v=2.910 \mu \mathrm{m} / \mathrm{s}$, and the gap between lens and iris is $5 \mu \mathrm{m} / \mathrm{s}$. Fig. 7 and 8 are flow characteristics of normal eyeball and shallow anterior chamber respectively.

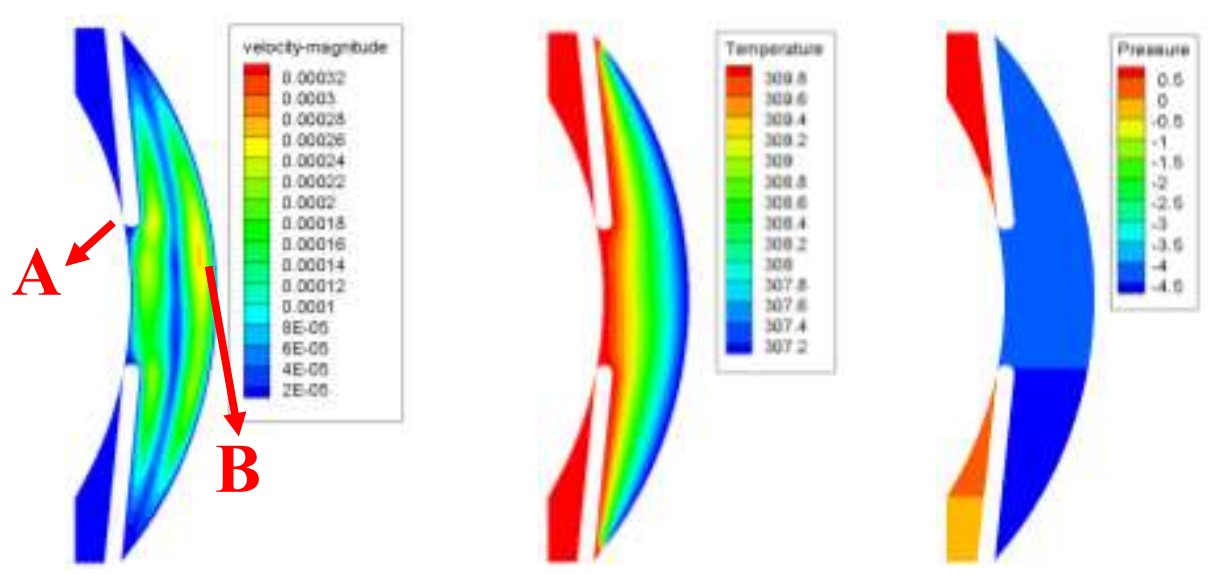

Fig. 8: the flow of aqueous humor (shallow anterior chamber).

The flow characteristics of shallow anterior chamber are similar to those of normal eyeball. The variation trends are the same, but the parameter values change greatly. The peak velocity of normal eye was $0.686 \mathrm{~mm} / \mathrm{s}$, while that of shallow anterior chamber was only $0.32 \mathrm{~mm} / \mathrm{s}$, while the velocity at the narrowest point of lens and iris remained unchanged. However, because of the low velocity of flow in shallow anterior chamber, there is less mixing and stirring to drive the flow in the area, the isotherm of the temperature field almost does not incline, and changes uniformly along the axis. And the pressure difference between the anterior chamber and the posterior chamber in shallow anterior chamber also increases to 5 $\mathrm{Pa}$. So when the depth decreases from $2.8 \mathrm{~mm}$ to $2.0 \mathrm{~mm}$, the maximum natural convection velocity decreases by one time, and the pressure difference between anterior and posterior chambers increases by $20 \%$. This is because when the temperature difference is constant, if the flow space of anterior chamber becomes smaller, the vortex generated by natural convection drive is affected by the wall, and there is not enough space for full development, resulting in a decrease in speed. According to the analysis, the geometric model of shallow anterior chamber depth is very important for the accuracy of glaucoma research. 


\subsection{Pupil block process}

Pupil block is the direct cause of elevated intraocular pressure in angle-closure glaucoma. In this part, the flow field characteristics of aqueous humor in different gaps (iris and lens) were analyzed to simulate the change of iris distance from lens, i.e. the pupil block process in the pathogenesis of primary angle closure glaucoma. In order to simulate the process of iris closing to the crystal in the process of pupil blockade, the mechanical parameters were simulated under five working conditions, i.e. the distance between iris and crystal is $10,5,2,1$ and $0.5 \mu \mathrm{m}$. Through the simulation during pupil block, we can better understand the pathogenesis of glaucoma and the characteristics of anterior chamber flow field, so as to improve the operation plan and avoid complications.

In this chapter, we use the physiological model of shallow anterior chamber to approach the real eyeball structure of glaucoma patients. The parameters used in the simulation are the same as the above. The changes of three parameters with the decrease of the gap are mainly studied: the difference of anterior and posterior chamber pressure, the velocity between iris and lens (point $\mathrm{A}$ in figure 8), and the maximum velocity in anterior chamber caused by natural convection (point $\mathrm{B}$ in figure 8).

From the comparative analysis in figs. 9 and 10, when the minimum gap between iris and lens is more than $5 \mu \mathrm{m}$, the difference of pressure is very small, which has no physiological significance to the eyeball tissue. When the iris lens gap continues to shrink, the pressure difference increases almost geometrically. When the distance is $0.5 \mu \mathrm{m}$, the pressure increases significantly, about 37 times of the pressure at $10 \mu \mathrm{m}$. It can be predicted that when the degree of pupil block increases, the gap between lens and iris decreases continuously, and aqueous humor can not flow through the pupil, the pressure difference will be thousands of times that of the normal pressure difference. This pressure difference will exert a forward push on the iris. The iris will rise forward, the anterior chamber angle closes, and the intraocular pressure will increase subsequently. The whole eyeball diffuse will cause damage to the retina of the fundus, and then change the visual field, eventually leading to blindness.

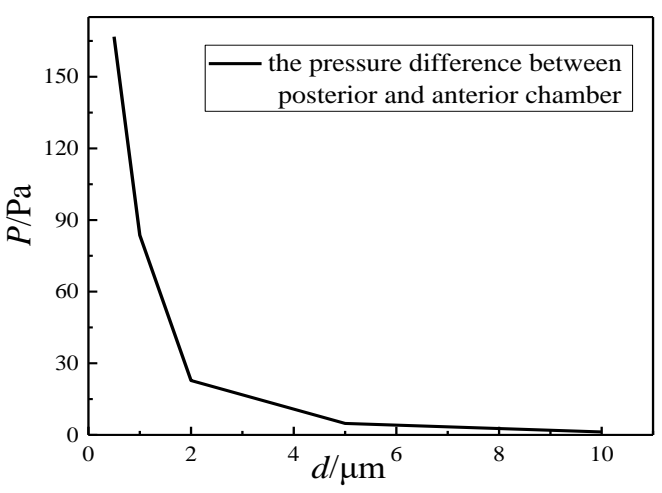

Fig. 9: the pressure difference.

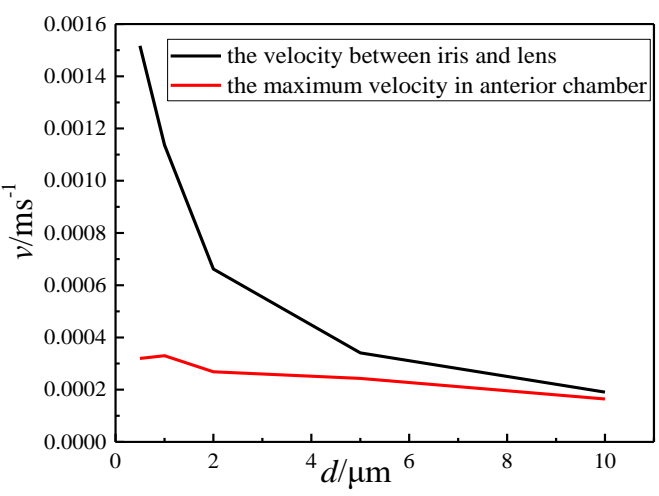

Fig. 10: the maximum velocity.

The velocity at the narrowest point (point A) increases sharply with the decrease of the spacing. Point B represents the intensity of natural convection. The general trend of the maximum velocity in the anterior chamber is gentle, but it increases slightly with the decrease of clearance. It should be the narrowest point where the velocity increases. The superposition of the flow field in the anterior chamber with natural convection results in a slight increase of the velocity.

\subsection{Improvement of parameters after operation}

This section simulates the flow characteristics after surgery to understand the improvement of the flow parameters and try to prevent the occurrence of complications by changing the operation parameters. When the LI window diameter was $0.56 \mathrm{~mm}$, the initial velocity through the LI window was set to $9.39 \mathrm{~mm} / \mathrm{s}$. According to this literature, the velocity entry conditions were set. The laser hole is at 12 o'clock and $5 \mathrm{~mm}$ from the pupil axis.

Fig. 11 shows the flow field of aqueous humor immediately after laser perforation. After laser perforation, the aqueous humor accumulated in the posterior enters the anterior chamber through the laser hole, effectively alleviating the high intraocular pressure caused by pupil block, and the intraocular pressure decreases from $\mathrm{kPa}$ to normal level (Pa). But the 
velocity near the laser hole is obviously increased, and the jet velocity produced is obviously higher than the maximum velocity in the anterior chamber when the iris is not perforated (increased by 40 times), thus affecting the flow of aqueous humor in the anterior chamber. Because the entrance velocity is two orders of magnitude larger than the natural convection velocity, there is no vortex caused by natural convection in the anterior chamber. At this time, the heat is transferred from the posterior part of the eye to the anterior part of the eye through thermal convection, which increases the temperature of aqueous humor near the laser hole.
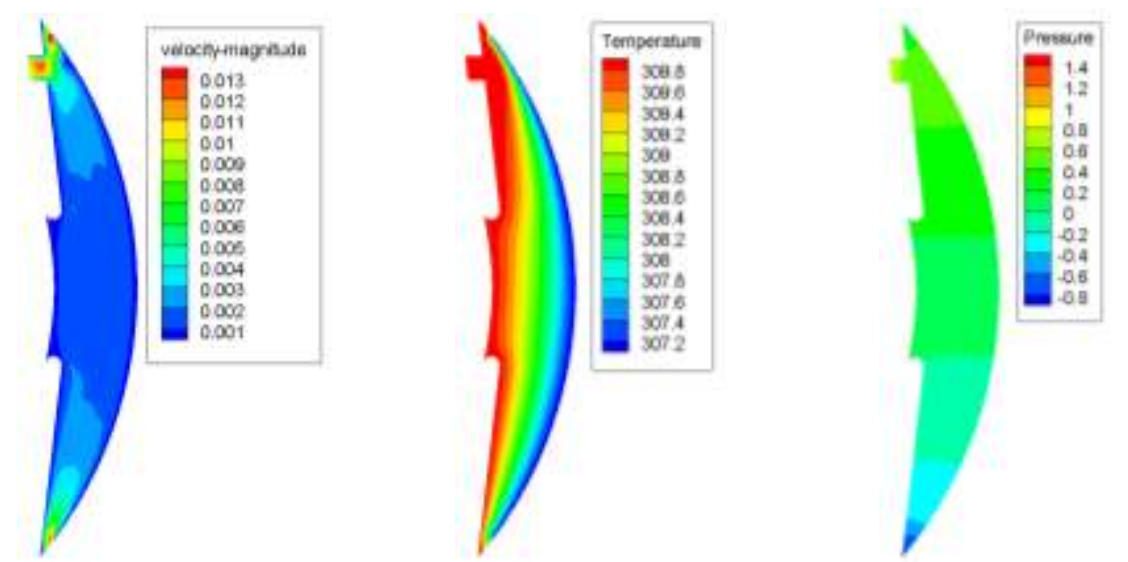

Fig. 11: Characteristics of aqueous humor after laser iridectomy.

\section{Conclusion}

In this paper, the main flow domain of aqueous humor was modelled by three kinds of structure of eyeball according to the physiological theory of the treatment process. They were normal eyeball, eyeball with the physiological structure of shallow anterior chamber depth and narrow angle and eyeball after laser iridotomy treatment. The finite volume method was used to discretize the computational model area. The flow of aqueous humor is simulated. The results showed that,

1)the geometric model of physiological structure of shallow anterior chamber depth was very important for theoretical study of glaucoma. When the depth of anterior chamber decreased from $2.8 \mathrm{~mm}$ to $2.0 \mathrm{~mm}$, the maximum velocity of natural convection doubled, and the pressure difference of shallow anterior chamber increased by $20 \%$.

2)Pupil block increases intraocular pressure sharply, which is equivalent to thousands of times the normal pressure, causing damage to intraocular tissues.

3)Laser iridectomy can effectively reduce the intraocular pressure caused by pupil block, but the velocity of aqueous humor after operation is 40 times of normal speed, and the increase of corneal shear stress leads to corneal damage.

\section{Acknowledgements}

This work was supported by the National Natural Science Foundation of China (51727811).

\section{References}

[1] Zhang, Shunhua, et al. "Optical Coherence Tomography Angiography of the Peripapillary Retina in Primary AngleClosure Glaucoma," American Journal of Ophthalmology, 2017.

[2] Jouzdani, Sara, R. Amini, and V. H. Barocas, "Contribution of Different Anatomical and Physiologic Factors to Iris Contour and Anterior Chamber Angle Changes During Pupil Dilation: Theoretical Analysis," Investigative Opthalmology \& Visual Science, vol. 54, no. 4, p. 2977, 2013.

[3] Feng, Zhang, C. Han, and D. O. Mechanics, "Numerical investigation of laser iridotomy influence on shear stress exerted on corneal endothelial cells," Journal of Medical Biomechanics, 2016.

[4] Abouali, Omid, et al. "Investigation of Saccadic Eye Movement Effects on the Fluid Dynamic in the Anterior Chamber," Journal of Biomechanical Engineering, vol. 134, no. 2, pp. 12-20, 2012.

[5] Ooi, E. H , and E. Y. K. Ng, "Effects of natural convection within the anterior chamber on the ocular heat transfer," International Journal for Numerical Methods in Biomedical Engineering, vol. 27, no. 3, pp. 408-423, 2015. 
[6] Yamamoto, Yasuaki, et al, "Effect of Anterior Chamber Depth on Shear Stress Exerted on Corneal Endothelial Cells by Altered Aqueous Flow after Laser Iridotomy," Investigative Opthalmology \& Visual Science, vol. 51, no. 4, pp. 1956, 2010.

[7] Avtar, R and R. Srivastava, "Aqueous outflow in Schlemm's canal," Applied Mathematics\s\&lscomputation, vol. 174, no. 1, pp. 316-328, 2014.

[8] Crowder, T. R., and V. J. Ervin, "Numerical simulations of fluid pressure in the human eye," Applied Mathematics and Computation, vol. 219, no. 24, pp. 11119-11133, 2013.

[9] Huang, E. C.and V. H. Barocas, "Accommodative microfluctuations and iris contour," Journal of Vision, vol. 6, no. 5, pp. 1-10, 2006.

[10] Wang, Wenjia, et al., "Fluid and structure coupling analysis of the interaction between aqueous humor and iris," BioMedical Engineering OnLine 15.2 Supplement, 2016.

[11] Yamamoto and Yasuaki, "Demonstration of Aqueous Streaming Through a Laser Iridotomy Window Against the Corneal Endothelium," Archives of Ophthalmology, vol. 124, no. 3, pp. 387-401, 2006.

[12] Heys, Jeffrey J., V. H. Barocas, and M. J. Taravella, "Modeling Passive Mechanical Interaction Between Aqueous Humor and Iris," Journal of Biomechanical Engineering, vol. 123, no. 6, pp. 540, 2001. 